\title{
ON A THEOREM OF MONTGOMERY AND SAMELSON
}

P. E. CONNER

In their paper on fibrations with singularities Montgomery and Samelson showed that if a compact connected Lie group acts differentiably and effectively on a sphere and if there is one stationary point, then the remaining orbits cannot all be of the same dimension [2]. In our note we will examine transformation groups in which all orbits have the same dimension. As a corollary, we shall extend the Montgomery-Samelson theorem to all closed simply connected manifolds. We shall not use a differentiability hypothesis.

We shall denote by $(L, X)$ the action of a compact, connected Lie group $L$ on a locally compact connected separable metric space $X$. It will be assumed that all the orbits of $(L, X)$ have the same dimension. We denote by

$$
m: L \times X \rightarrow X
$$

the function defining the action of $L$ on $X$, and by

$$
\eta: X \rightarrow X / L
$$

the natural map of $X$ onto the orbit space $X / L$. Let $G_{x}, H_{x}$ and $N_{x}$ respectively denote the isotropy group at $x$, the identity component of $G_{x}$, and the normalizer in $L$ of $H_{x}$. Since all the orbits in $X$ have the same dimension, $\operatorname{dim} H_{x}=\operatorname{dim} H_{y}$. If $x$ and $y$ are sufficiently close there is an element $g \in L$ such that $g G_{x} g^{-1} \subset G_{y}[3$, p. 215]. Now $\operatorname{dim} H_{x}=\operatorname{dim} H_{y}$, so we must have $g H_{x} g^{-1}=H_{y}$. Since $X$ is connected, this implies that all the $H_{x}$ are conjugate.

Let $F_{x} \subset X$ be the set of stationary points of $H_{x}$. We first observe that if $F_{x} \cap F_{y} \neq \phi$, then $H_{x}=H_{y}$, for if not, then the points of the intersection are stationary under the closed subgroup generated by $H_{x}$ and $H_{y}$, but the identity component of this subgroup will contain $H_{x} \cup H_{y}$ so that it cannot possibly be conjugate to $H_{x}$. If $g \in L$ is such that $g F_{x}=F_{x}$, then $g \in N_{x}$, for $g F_{x}$ is the set of stationary points of $g H_{x} g^{-1}$.

Lemma 1. For any $x \in X$, the orbit space $F_{x} / N_{x}$ is homeomorphic to $X / L$.

We may use the map $\eta$ to define a map

$$
\eta: F_{x} / N_{x} \rightarrow X / L \text {. }
$$

Received by the editors December 12, 1957. 
The map is onto, for if $O(y) \subset X$ is the orbit of $y$, then $g y \in F_{x}$, where $g \in L$ is such that $g H_{y} g^{-1}=H_{x}$. The map is 1-1 since $g F_{x}=F_{x}$ implies that $g \in N_{x}$. We omit the proof that the inverse is continuous.

Let $\widetilde{F}_{x}$ be a component of $F_{x}$ and let $B_{x} \subset N_{x}$ be the subgroup of $L$ which maps $\tilde{F}_{x}$ onto itself. We define two mappings

$$
\begin{aligned}
& \nu_{1}: X \rightarrow L / B_{x}, \\
& \nu_{2}: X \rightarrow L / N_{x} .
\end{aligned}
$$

The first map is defined by sending the points of $g \widetilde{F}_{x}$ into the coset $g B_{x}$, and the second by sending points of $g F_{x}$ into the coset $g N_{x}$.

LeMma 2. The mappings $\nu_{1}$ and $\nu_{2}$ define local product bundles with fibre and group $\tilde{F}_{x}, B_{x}$ and $F_{x}, N_{x}$ respectively.

It is well known that

$$
\nu: L \rightarrow L / B_{x}
$$

is a principal bundle. Let $J \subset L$ be a local cross-section at the identity $e$ of $\nu$. The cell $J$ has the property that if $g, h \in J$ and $g^{-1} h \in B_{x}$, then $g^{-1} h=e$.

The map

$$
m: J \times \widetilde{F}_{x} \rightarrow X
$$

is a homeomorphism, for if $g y=h z$, then $g^{-1} h z=y$, and $g^{-1} h \in B_{x}$, which implies that $g=h$ and $y=z$. Of course $\nu_{1}\left(J \tilde{F}_{x}\right)=\nu(J)$. The translates of $J$ define a set of neighborhoods on $L / B_{x}$ which serve at once as co-ordinate neighborhoods for $\left[L, L / B_{x}, B_{x} ; \nu\right]$ and for $\left[X, L / B_{x}, \widetilde{F}_{x} ; \nu_{1}\right]$, and this determines the co-ordinate transformations of $\left[X, L / B_{x}, \widetilde{F}_{x} ; \nu_{1}\right]$. A similar statement applies to $\left[X, L / N_{x}\right.$, $\left.F_{x} ; \nu_{2}\right]$.

It should be observed that if $y \in \widetilde{F}_{x}$, then $G_{y} \subset B_{x}$. It might be noted in passing that some local properties of $\widetilde{F}_{x}$ can be determined now. For example, if $X$ is an ANR, so is $\widetilde{F}_{x}$, or if $X$ is a manifold, $\widetilde{F}_{x}$ is a generalized manifold in the sense of local homology properties.

Lemma 3. If for the transformation group $(L, X)$ the space $X$ is simply connected, and if all the orbits have the same dimension, then $B_{x}$ is connected.

The proof is immediate upon examining the fiber bundle $\left\lfloor X, L / B_{x}, \tilde{F}_{x} ; \nu_{1}\right]$. Since $X$ is simply connected, $\pi_{1}\left(L / B_{x}\right)=0$, but on the other hand, there is a homomorphism of $\pi_{1}\left(L / B_{x}\right)$ onto $B_{x} / B_{x}^{c}$, where $B_{x}^{c}$ is the identity component of $B_{x}$.

THEOREM 1. If $(L, X)$ denotes the action of a compact, connected Lie 
group on a compact simply connected $A N R$ such that $\chi(X) \neq 0$, and if all the orbits have the same dimension, then all the isotropy groups are connected and conjugate.

We may take $L$ to be simply connected. Since $\chi(X) \neq 0$, any maximal torus in $L$ has a stationary point in $X$, hence $H_{x}$ is a subgroup of maximum rank. By the preceding lemma $B_{x}$ is connected and $H_{x} \subset B_{x} \subset N_{x}$, but $N_{x} / H_{x}$ is finite, so $B_{x}=H_{x}$. Finally, since $G_{x} \subset B_{x}$, $G_{x}=H_{x}$. This means that $L$ defines a fibration of $X$ with fibre $L / H_{x}$ [3].

THEOREM 2. If $\left(L, M^{n}\right)$ denotes the effective action of a compact, connected Lie group on a closed simply connected manifold, and if there is a stationary point, then the remaining orbits cannot all have the same dimension.

Let us suppose that the theorem is false, and that $\left(L, M^{n}\right)$ is a counter example. We consider the transformation group $\left(L, W^{n}\right)$ on the open simply connected manifold $W^{n}$ obtained by deleting the stationary point from $M^{n}$. We assume that the orbits of $\left(L, W^{n}\right)$ all have the same dimension. It was shown in [1] that any maximal torus in $L$ has a stationary point on $W^{n}$, thus $H_{x}$ has maximal rank. Furthermore $\pi_{1}\left(L / N_{x}\right) \simeq N_{x} / H_{x}$, so that $F_{x}$ has $\left(N_{x} / H_{x}\right)$ components. Since $N_{x}$ must act transitively on the components of $F_{x}$, it follows that $B_{x}=H_{x}$. This implies that $G_{x}=H_{x}$, so all the isotrophy groups are connected and conjugate. Thus $\left(L, W^{n}\right)$ defines a compact fibration of $W^{n}$ by $L / I I_{x}$ which must be proper, and this contradicts [1].1

This is the extension of the Montgomery-Samelson theorem referred to in our title. It should be kept in mind that there is a conjecture to the effect that a compact connected Lie group acting on a sphere with one stationary point must have another. The Montgomery-Samelson theorem supports this conjecture and is a step toward a solution.

\section{REFERENCES}

1. P. E. Conner, On the impossibility of fibering certain manifolds by a compact fiber, Michigan Mathematical Journal (to appear).

2. D. Montgomery and H. Samelson, Fiberings with singularities, Duke Math. J. vol. 13 (1946) pp. 51-56.

3. D. Montgomery and L. Zippin, Topological transformation groups, Interscience, 1955.

\section{UNiversity of Michigan}

1 The principal result of [1] asserts that an open simply connected manifold whose one-point compactification is again a manifold cannot be fibred by a compact fibre. 